\title{
Nanogratings Formation in a System of Ultra-short Laser Pulses - Metalloorganic Gas - Deposited Metal - Sapphire in Sinergetic Interference Field with Waveguide Modes Participation
}

\section{V.S. MAKIN and R.S. MAKIN}

Laser Physics Department, Scientific Research Institute for Optoelectronic Instruments Engineering, Sosnovy Bor, Leningrad region, Russia

Department for nuclear reactors and materials, National Research Nuclear University MEPhl (Moscow Engineering Physics Institute), Kashirskoe shosse 31, Moscow, 115409, Russia

\section{Abstract}

Experimental results of laser-induced deposition of structured metal films from

Corresponding Author: V.S. MAKIN

makinvs@niioep.ru

Received: 28 January 2018

Accepted: 15 March 2018

Published: 25 April 2018

Publishing services provided by Knowledge $\mathrm{E}$

(c) V.S. MAKIN and R.S.

MAKIN. This article is distributed under the terms of the

Commons Attribution License,

which permits unrestricted use and redistribution provided that the original author and source are credited.

Selection and Peer-review under the responsibility of the PhIO Conference Committee. metalloorganic vapor have been analyzed. A new physical model has been put forward to explain the results. The model is based on the interference phenomena between the incident laser radiation and waveguide modes of a thermally induced gradient waveguide in the substrate.

Keywords: femtosecond radiation, metal deposition, interference, waveguide modes, surface nanogratings.

\section{Introduction}

The problem of elucidation of physical mechanisms of micro- and nanostructures formation under the interaction of ultrashort intensive laser radiation with condensed media is of current interest. Nanostructures in the form of metal films oriented by laser radiation polarization and deposited onto a dielectric substrate at sufficiently lower power densities ( $\sim 20 \mathrm{~mW})$ and high pulse repetition rate $(\mathrm{f}=80 \mathrm{MHz})$ were produced in experiments [1-3]. The deposited gratings $(\mathbf{g})$ have orientation $\mathbf{g} \perp \mathbf{E}$ and period $\mathrm{d}<\lambda$, where $\mathbf{E}$ is the electric field strength vector and $\lambda=400 \mathrm{~nm}$ is the wavelength of the incident radiation. Deposition of tungsten from the tungsten hexacarbonil vapor $\mathrm{W}(\mathrm{CO})_{6}$ in the experiments was initiated by the linearly polarized laser radiation ( $\lambda=400$ $\mathrm{nm}, \tau=90 \mathrm{fs}, \mathrm{f}=80 \mathrm{MHz}, \mathrm{v}=(0.2 \div 1.0) \mu \mathrm{m})$. Here, $\mathrm{v}$ is the scanning velocity of laser radiation over the substrate. High pulse rate $(f=80 \mathrm{MHz})$ of the laser radiation is of 
importance for the further investigation. The obtained results [1-3] were not explained. The authors suggested that the effect might be related to the interference of incident radiation and surface plasmon polaritons induced by it [1].

\section{Physical model and discussion}

We suggest a novel model to describe the formation process of regular nanopatterns with normal orientation. Here orientation of the nanogratings is associated with conclusions made from the universal polariton model (UPM) of laser-induced damage of condensed matter [4] aimed to form nanostructures caused by the interference with the surface plasmon polaritons. In our model we took into account the peculiarities of the process under consideration: high repetition rate of laser pulses ( $80 \mathrm{MHz}$ ) and sapphire substrates. Absorption of the incident laser radiation in the surface layer of the sapphire substrate results in heating of the surface layer and formation of the spatially localized gradient waveguide structure because for sapphire the value of $\mathrm{dn} / \mathrm{dT}$ is positive and $\mathrm{dn} / \mathrm{dT}=1.4110^{-3} \mathrm{~K}^{-1}$ for $\lambda=400 \mathrm{~nm}$ [5]. The gradient waveguide structures and formation of surface micrograting under nanosecond laser pulses for mono crystalline silicon and germanium have been thoroughly studied in our work [6]. The incident laser radiation interferes with the waveguide mode excited by it because both waves are coherent. Total distribution of the electromagnetic field intensity is generated in the form of a standing wave. Modes with the highest Q factors of these gradient waveguides are TE type modes. It follows that the resulting interference grating has $\mathbf{g} \perp \mathbf{E}$ orientation (see Fig.1). In the process of the laser radiation scanning the dynamic waveguide moves with the laser spot. The deposition rate of tungsten depends on distribution of the total electromagnetic field intensity and it is higher at the maximum peaks of the intensity. It is obvious that in interference the positive feedback by amplitude of the resulting grating arises and leads to formation of a metal grating with strictly defined periods on the substrate surface. The area of the possible spatial periods (d) of the produced gratings is described by the following inequalities:

$$
\frac{1}{n+\frac{d n}{d T} \Delta T} \leq \frac{d}{\lambda} \leq \frac{1}{n}
$$

where $\Delta n=d n / d T \Delta T, n$ is the refractive index of substrate, $d n / d T$ is the temperature derivative of the refractive index for given $\lambda, \Delta T$ is the increase of substrate surface temperature. For sapphire and wavelength of $\lambda=400 \mathrm{~nm}$ the value of the refractive index is $n=1.78$ [5]. From (1) it follows that with the decrease of the value of $\Delta T$ there is the tendency to increase of period $d$ and it verges to the maximum value $d=\lambda / n$. The 
tendency is confirmed by the experimental data shown in Fig. 2: the period is higher for a) higher scanning speeds (low $\Delta \mathrm{T}$ ) and b) low power densities of laser radiation (low $\Delta \mathrm{T}$ ).

In case the electric field vector is oriented $\mathbf{E} \perp \mathbf{s}$ the excited TE type waveguide modes propagate in direction of vector, $\mathbf{k}_{s} \| \mathbf{s}$, and coherent translation of the relief is observed along $\mathbf{s}$ direction and high quality nanogratings are formed. Formation of the grating takes place in the self-consistent interference field (see Fig.1A, B). In other words, the deposited metal film "visualizes" the intensity distribution of the standing wave. In case when the waveguide modes

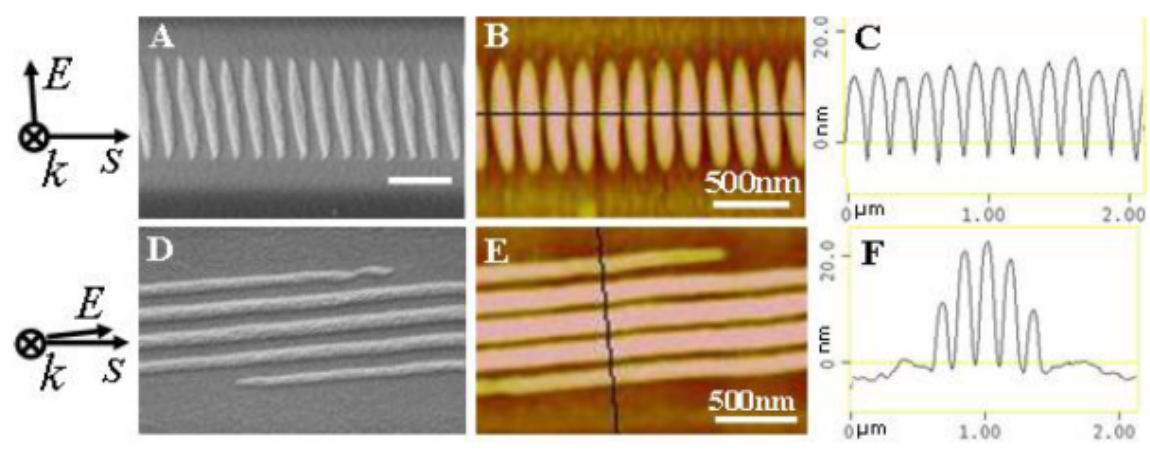

Figure 1: SEM images of $(A)$ gratings with $\mathbf{g} \| \mathbf{s}$ and $(B)$ with $\mathbf{g} \perp \mathbf{s}$ on sapphire at $P=22 \mathrm{~mW}$ and $\mathbf{v}=0.8$ $\mu \mathrm{m} / \mathrm{s}$. (B-C) and (E-F) are the corresponding AFM images and cross-sectional profiles for (A) and (D), respectively. In the insets: $\mathbf{k}, \mathbf{E}$, and $\mathbf{s}$ indicate the relative orientation of laser radiation, electric field strength, and substrate scanning of laser radiation, respectively [3].

propagate along orthogonal direction $\mathbf{E} \| \mathbf{s}, \mathbf{k}_{s} \perp \mathbf{s}$ (see Fig.1D,E) the energy of waveguide modes is partially carried out of the area where the structures are forming. The fact that maximal thickness of the deposited film does not exceed $16 \mathrm{~nm}<\delta$ (see Fig.1C, F) and the film is partially transparent for laser radiation confirms the proposed model. Here $\delta$ is the skin-depth of tungsten film, and $\mathbf{k}_{s}$ is the wave vector of waveguide modes.

Close inspection of images of the gratings being formed reveals small-scale gratings $\mathbf{G}|| \mathbf{E}$ with period $\mathrm{D} \approx 25 \mathrm{~nm}$ located on the ridges of the main relief $(\mathbf{g})$ [1]. The period of this grating can be described in the framework of a nonlinear mathematical model for laser-induced formation of spatial periods of gratings and $D=\lambda / 16 \xi[7]$, where $\xi$ is the real part of refractive index for WSP of sapphire-tungsten-vacuum boundary [8]. Formerly the similar gratings with abnormal orientation were revealed on the condensed matter surfaces with essentially different physical properties: metals, semiconductors and dielectrics under the interaction with ultrashort and nanosecond laser pulses $[8,9]$. The physical model, which describes their formation, includes excitation and interference of the channel surface plasmon polaritons (CSP) or wedge surface plasmon 
polaritons with opposite propagation directions [8]. Valleys (CSP) or ridges (WSP) of the main relief are driving structures for these waves. In routine experiments (e.g. with linearly polarized laser radiation) the excitation of CSP (WSP) is difficult to realize due to lack of electric field component necessary for the excitation along its propagation direction. In our case the situation is favorable for CSP (WSP) excitation because $\mathbf{E}|| \mathbf{k}$, where $\mathbf{k}$ is the wavevector of CSP (WSP). One of the obstacles is the efficient thermal smoothing of the small-scale relief grating since $\mathrm{D} \leq 30 \mathrm{~nm}$. This is the case in which $\mathrm{I}_{p h}$ $\geq D$, where $\mathrm{I}_{p h}$ is the mean free path of a phonon and the ordinary macroscopic theory of thermal conductivity is not applicable. Scattering of phonons by grating $\mathbf{G}$ leads to the additional spatially structured dissipation of energy. Probably, the additional energy losses may be associated with the recently revealed non-exponential attenuation of the surface plasmon polaritons in metals [10] that can result in increased losses in metal during CSP (WSP) propagation. This superanomalous skin effect of the surface plasmon polaritons in metals is conditioned by non-locality and singularity of dielectric permittivity of metals. Note that anomalous nanogratings with period $D=20 \mathrm{~nm}$ have been experimentally found out on surface of molybdenum, which is close to tungsten by its properties, under irradiation by a set of linear polarized femtosecond laser pulses $(\lambda=800 \mathrm{~nm})[11]$.

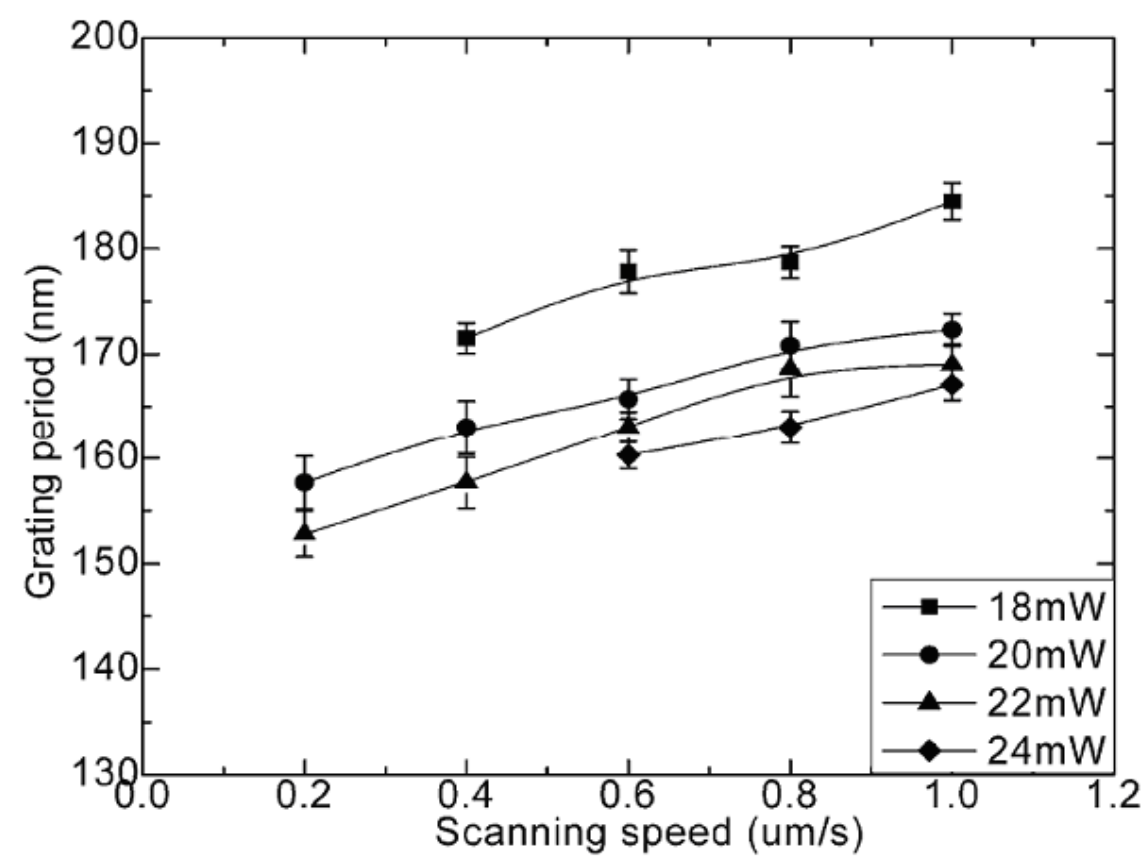

Figure 2: Plot of dependence of grating period on scanning speed for different laser power [1]. 


\section{Conclusion}

The physical model is proposed to explain formation of the metal gratings as a result of the metalloorganic vapor decomposition with deposition onto the sapphire substrate under the action of linearly polarized femtosecond laser radiation with high pulse rate and low average power density. The model involves development of a thermal gradient waveguide in the surface layer of sapphire, WM and incident laser radiation interference with generation of standing waves. Hence the inhomogeneous metal deposition does not depend on the initial spectrum of the substrate surface nanoroughness but is determined by the self-organized structure of the interference field in the near field of the substrate surface. This ensures high quality of the resulting nanogratings. Gratings with abnormal orientation and small period of $d=25 \mathrm{~nm}$ were found out on the SEM images of the deposited films. Their formation is explained as the result of mutual interference of the wedge surface plasmon polaritons excited by laser radiation.

\section{References}

[1] Tang M., Zhang H., Her T.-H. Self-assembly of tunable and highly uniform tungsten nanogratings induced by a femtosecond laser with nanojoule energy. Nanotechnology. V.18. P. 485304 (2007).

[2] H. Zhang, M. Tang, J. McCoy, T.-H. Her. Deposition of tungsten nanogratings induced by a single femtosecond laser pulse. Optics Express. V. 15. No 10. P. 5937 (2007).

[3] M. Tang, H. Zhang, T.-H. Her. Observation of self-assembled periodic nanostructures in both ablation and deposition regimes. Proceedings of SPIE. V. 6879. P. 68791-1 (2008).

[4] Makin V.S., Makin R.S. Nonlinear interaction of linear polarized laser radiation with condensed media and diffraction limit overcoming. Optics and Spectroscopy. V.112. No 2. P. $193-198$ (2012).

[5] Acoustic Crystals. Ed. By S.V. Koryshev. M.: Nauka. 1982. 632 p.

[6] Makin V.S., Pestov Yu.I., Privalov V.E. Thermal waveguide and fine-scale periodic relief on the semiconductor's surface induced by TEA $\mathrm{CO}_{2}$ laser radiation. Optical Memory and Neural Network Modeling. No1. Pp52-61 (2012).

[7] Makin V.S., Makin R.S. Feigenbaum's universality and Sharkovsky order in laserinduced periodic structures on surfaces and in bulk of condensed media. P.302322. in "Nonlinearity in modern nature" (Synergy: from past to future). Ed. by G.G. 
Malinetski. LKI Publisher. 2009. 424 P.

[8] Makin V.S., Logacheva E.I., Makin R.S. Localized surface plasmon polaritons and nonlinear overcoming of the diffraction optical limit. Optics and Spectroscopy.V. 120. No 4. P.610-614 (2016).

[9] Makin V.S., Pestov Yu. I., Makin R.S. Abnormal spatial nanogratings formation by long pulse laser radiation on condensed matter surfaces. Proceedings of International Conference "Days on Diffraction". Pp. 245-250 (2016).

[10] Larkin I.A., Keil K., Vagov A., Croitoru M.D., Axt V.M. Superanomalous skin effect for surface plasmon polaritons. Phys. Rev. Lett. V. 119. P. 176801 (2017).

[11] M.D. Dar, N.A. Saad, C. Sahoo, S.R.J. Naraharisetty, N.R. Desai. Ultrafast laser-induced reproducible nano-grating on a molybdenum surface. Laser Physics. V. 14. P. 026101 (2017). 